\title{
NGHIÊN CỨU BÊ̂NH MẠCH MÁU HẮC MẠC DẠNG POLYP BẰNG CHỤP MACH OCT TẠI BÊ̂NH VIỆN LÃO KHOA TRUNG ƯƠNG
}

\begin{abstract}
TÓM TẮT.
Mục tiêu: Mô tả bệnh mạch máu hắc mạc dạng polyp bằng chụp mạch OCT. Đối tượng và phưởng pháp nghiên cứu: nghiên cứu mô tả cắt ngang trên bệnh nhân được chẩn đoán PCV bằng chụp ICGA, tại khoa mắt Bệnh viện Lão khoa Trung ương từ 8/2020 đến $8 / 2021$. Kết quả: nghiên cứu 28 bênh nhân (28 mắt) được chẩn đoán xác định trên chụp xanh indocyanine (ICG), trong đó có 17 bệnh nhân (BN) nam, 11 bệnh nhân nữ, tuổi trung bình $61,07 \pm 8,41$ (cao nhất 80 tuổi, thấp nhất 44 tuổi). ICGA phát hiện PL ở tất cả các mắt $(100 \%)$, trong khi OCTA phát hiển $\mathrm{PL}$ ở 17 mắt $(60,7 \%)$; ICGA phát hiện $\mathrm{BVN}$ ở 14 mắt (50\%), trong khi OCTA phát hiện BVN ở 16 mắt $(57,1 \%)$. Tất cả các BVN được OCTA phát hiện đều nằm giữa RPE và màng Bruch. Diện tích polyp trung bình trong ICGA và OCTA $($ OCTA + và ICGA +) là 0,47 $\pm 0,31 \mathrm{~mm}^{2}$ và $0,17 \pm 0,16 \mathrm{~mm}^{2}$. Kết luân: ICG là tiêu chuẩn vàng trong chẩn đoán PCV, sử dụng OCTA mà không tiêm thuốc nhuộm có thể hữu ích để theo dõi sự tiến triển hoăc tái phát. Diên tích polyp trung bình được đo bằng ICGA lớn hơn đáng kê so với đo bằng OCTA.
\end{abstract}

Tư khóa: Bênh mạch máu hắc mac dang polyp, chụp xanh indocyanine, chụp cắt lớp vỗng mạc, chụp cắt lớp quang học mạch máu.

\section{SUMMARY}

\section{CHARACTERISTICS OF POLYPOIDAL} \section{CHOROIDAL VASCULOPATHY EVALUATED BY OPTICAL COHERENCE TOMOGRAPHY ANGIOGRAPHY}

Purpose: Characteristics of Polypoidal Choroidal Vasculopathy (PCV) evaluated by Optical coherence tomography angiography. Materials and methods: analysis all the patients diagnosed PCV in ophthalmology department of National Geriatric Hospital from 8/2020 - 8/2021. Results: Twenty eight eyes of 28 patients ( 17 male, 11 female) were verified in ICG. Average age was $61,07 \pm 8,41$ years (max 80 years, min 44 years). ICGA detected PL in all eyes $(100 \%)$, whereas OCTA detected PL in 17 eyes $(60,7 \%)$; ICGA detected BVN in 14 eyes (50\%), whereas OCTA detected BVN in 16 eyes (57,1\%). All of the BVNs detected by OCTA were located between

${ }^{1}$ Trường Đại học Y dược Thái Bình,

${ }^{2}$ Hội Whãn khoa Viêt Nam,

3 Trường Đại học Y Hà Nối,

${ }^{4}$ Trường Đại hoc Y Dược Thái Nguyên

Chịu trách nhiệm chính: Nguyễn Thị Thu Trang

Email: trangnguyen.yk@gmail.com

Ngày nhận bài: 10.9.2021

Ngày phản biên khoa hoc: 26.10.2021

Ngày duyệt bài: 12.11.2021

\section{Nguyễn Thị Thu Trang1, Hoàng Thị Phúc ${ }^{2}$, Mai Quốc Tùng ${ }^{3}$, Lương Thị Hải Hà ${ }^{4}$}

the RPE and Bruch's membrane. The mean PA in ICGA and OCTA (OCTA+ and ICGA+) was $0,47 \pm 0.31 \mathrm{~mm}^{2}$ and $0.17 \pm 0.16 \mathrm{~mm}^{2}$. Conclusion: ICG is the gold standard in the diagnosis of PCV, using OCTA without dye injection may be useful to monitor progression or recurrence. OCTA might detect more BVNs and fewer PLs compared with ICGA, and PL detected by OCTA might be smaller than those detected by ICGA.

Keywword: Polypoidal choroidal vasculopathy, indocyanine green angiography, Spectral-domain OCT, Optical coherence tomography angiography.

\section{I. ĐĂT VẤN ĐỀ}

Bệnh mạch máu hắc mạc dạng polyp (Polypoidal choroidal vasculopathy - PCV) là bệnh lý gây nên do sự giãn mạch dạng polyp và chia nhánh bất thường mạng mạch máu hắc mạc. Bệnh được miêu tả lần đầu tiển đầu những năm 1982 bởi tác giả Yannuzzi với thuật ngữ "bệnh polyp hắc mạc vô căn"1.

Chụp mạch ICG là khám nghiệm cận lâm sàng kinh điển, tiêu chuẩn vàng để chẩn đoán bệnh mạch máu võng mạc và hắc mạc, đặc biệt là trong chẩn đoán hình ảnh polyp hắc mạc. Trên chụp OCT, PCV gây bong và nâng lớp BMST, dấu hiệu khuyết BMST, mạng mạch nuôi có thể được nhận biết bởi dấu hiệu hai lớp 2,3.

Chụp mạch OCT (OCTA) là phương pháp không xâm lấn, không sử dụng thuốc nhuộm, thời gian thu nhận hình ảnh ngắn. OCTA có hiệu quả trong việc phát hiện PCV, mạng mạch máu phân nhánh $(\mathrm{BVN})$, tỷ lệ phát hiện polyp trên OCTA thay đổi từ $17 \%$ đển $85 \% 4$.

Ở Việt Nam, hiện chưa có đề tài nào nghiên cứu về chụp mạch OCT trong bệnh PCV. Do đó chúng tôi thực hiện đề tài nhẳm mô tả bệnh mạch máu hắc mạc dạng polyp bằng chụp mạch OCT và đối chiếu đặc điểm bệnh mạch máu hắc mạc dạng polyp trên chụp mạch OCT với chụp OCT cắt lớp và chụp ICG.

\section{II. ĐỐI TƯỢNG VÀ PHƯƠNG PHÁP NGHIÊN CỨU \\ 2.1. Đối tượng nghiên cứu \\ *Đối tượng và thời gian nghiên cứu:} Nghiên cứu được tiến hành trên những bệnh nhân được chẩn đoán là bệnh mạch máu hắc mạc dạng polyp đến khám và điêu trị tại Khoa mắt Bệnh viện Lão khoa Trung ương từtháng $8 / 2020$ đến tháng 8/2021.

*Tiêu chuẩn lựa chọn: Những bệnh nhân 
trong đối tượng nghiên cứu phải đủ điều kiện là đã được chẩn đoán xác định bệnh mạch máu hắc mạc dạng polypvà đồng ý tham gia nghiên cứu.

*Tiêu chuẩn loại trừ:

- Đục các môi trường của mắt.

- Không đồng ý tham gia nghiên cứu.

- Già yếu, phụ nữ mang thai.

2.2. Phương pháp nghiên cứu: Nghiên cứu mô tả tiến cứu.

*Phương tiện. Bảng đo thị lực Logmar và hộp kính, nhãn áp kế Maclakov, sinh hiển vi khám bệnh và máy chụp ảnh đáy mắt, kính soi mắt Volk super field, máy chụp OCTA Carl Zeiss (Cirrus HD - OCT 5000 AngioPlex), thuốc giãn đồng tử Mydrin P0,5\%.

*Các bước tiến hành

- Hỏi bệnh: khai thác triệu chứng chủ quan: nhìn mờ, nhìn méo hình, ám điểm, thay đổi màu sắc, khai thác tiền sử bệnh về mắt, các bệnh lý toàn thân: cao huyết áp, đái tháo đường, mõ máu...

- Khám: đo thị lực, đo nhãn áp, khám bán phần trước bằng sinh hiển vi loại trừ bệnh lý bán phần trước, khám soi đáy mắt với đồng tử giãn tốt đánh giá dịch kính, võng mạc (xuất huyết, xuất tiết, nốt màu đỏ cam, bong thanh dịch, bong biểu mô sắc tố, phù hoàng điểm, sắc tố, sẹo xơ võng mạc, drusen...)

- Chẩn đoán hình ảnh: Tất cả các bệnh nhân trong nghiên cứu đều được: chụp OCT và chụp ICGA.

*Xử lý số liệu:chúng tôi xử lý số liệu bằng phần mềm SPSS 16.0.

\section{KẾT QUẢ NGHIÊN CứU}

Bảng 1. Triệu chứng cơ năng

\begin{tabular}{|c|c|c|}
\hline Triệu chứng cơ năng & $\mathbf{n}$ & $\mathbf{\%}$ \\
\hline Nhìn mờ & 26 & 92,9 \\
\hline Nhìn méo hình & 14 & 50 \\
\hline Amm điếm & 17 & 60,7 \\
\hline Thay đối màu sắc & 4 & 14,3 \\
\hline Nhìn hình thu nhỏ lại & 14 & 50 \\
\hline Triệu chứng khác & 2 & 7,1 \\
\hline
\end{tabular}

Triệu chứng nhìn mờ gặp hầu hết các trường hợp tương ứng 26 mắt (chiếm 92,9\%). Ngoài ra bệnh nhân có các dấu hiệu cơ năng khác của hội chứng hoàng điểm như ám điểm $(60,7 \%)$, nhìn méo hình $(50 \%)$, thay đổi màu sắc $(14,3 \%)$ và nhìn hình nhỏ lại (50\%). Có 2 bệnh nhân bị triệu chứng khác là đau nhức mắt.

Bảng 2. Các dâu hiệu thực thể trên lâm sàng

\begin{tabular}{|c|c|}
\hline Dấu hiệu thực thế & $\mathbf{n}(\%)$ \\
\hline Drusen cứng & $19(67,9)$ \\
\hline Drusen mềm & $12(42,9)$ \\
\hline Phù hoàng điếm & $20(71,4)$ \\
\hline
\end{tabular}

\begin{tabular}{|c|c|}
\hline Biến đối biếu mô sắc tố & $20(71,4)$ \\
\hline Bong biếu mô sắc tố & $22(78,6)$ \\
\hline Tân mạch & $6(21,4)$ \\
\hline Bong thanh dịch võng mạc & $20(71,4)$ \\
\hline Sẹo xơ hoàng điểm & $5(17,9)$ \\
\hline Trong nhóm bênh nhân nghiên cứu có 20 mắt
\end{tabular}

Trong nhóm bệnh nhân nghiên cứu có 20 mắt phù hoàng điểm dạng nang chiếm $71,4 \%$, bong thanh dịch gặp ở 20 mắt $(71,4 \%)$. Triệu chứng gặp nhiều nhất là bong biểu mô sắc tố chiếm 78,6\% (22 mắt). Trên lâm sàng có 19 mắt chiếm $67,9 \%$ có biểu hiện drusen.

Bảng 3. Mạng lưới mạch máu chia nhánh bât thường (BVN) trên OCTA

\begin{tabular}{|c|c|c|c|}
\hline BVN & $\begin{array}{c}\text { Phân loại } \\
\text { BVN }\end{array}$ & $\mathbf{n}$ & $\%$ \\
\hline \multirow{3}{*}{$\begin{array}{l}\text { Có quan } \\
\text { sát thây } \\
\text { trên OCTA }\end{array}$} & Loại 1 & 4 & 14,3 \\
\hline & Loại 2 & 8 & 28,6 \\
\hline & Loại 3 & 4 & 14,3 \\
\hline \multicolumn{2}{|c|}{$\begin{array}{c}\text { Không quan sát thấy } \\
\text { trên OCTA }\end{array}$} & 12 & 42,9 \\
\hline \multicolumn{2}{|c|}{ Tống } & 28 & 100 \\
\hline
\end{tabular}

Chúng tôi quan sát BVN trên OCTA nhận thây có 16 trường hợp phát hiên được BVN trền hình ảnh chụp $(57,1 \%)$ và 12 trường hợp không thấy BVN (42,9\%). Các hình thái BVN quan sát được lần lượt: $14,3 \%$ loại $1,28,6 \%$ loại 2 và $14,3 \%$ loại 3 .

Bảng 4. Quan sát polyp và BVN trên OCTA

\begin{tabular}{|c|c|c|}
\hline BVN OCTA & Không polyp & Có polyp \\
\cline { 2 - 3 } & $\mathbf{n}(\%)$ & $\mathbf{n}(\%)$ \\
\hline $\begin{array}{c}\text { Không quan sát } \\
\text { thấy }\end{array}$ & $8(66,7)$ & $4(33,3)$ \\
\hline Loại 1 & $1(1 / 4)$ & $3(3 / 4)$ \\
\hline Loại 2 & 0 & $8(8 / 8)$ \\
\hline Loại 3 & $2(2 / 4)$ & $2(2 / 4)$ \\
\hline
\end{tabular}

Quan sát polyp và BVN trên OCTA nhận thây: 12 trường hợp không quan sát được $B V N$, trên OCTA thấy 8 mắt không có polyp (chiếm $66,7 \%$ ) và 4 mắt có nhìn thấy polyp (chiếm $33,3 \%$ ). Với 4 trường hợp BVN loại 1 có 3 trường hợp phát hiện polyp. BVN loại 2 hầu như quan sát thấy polyp và BVN loại 3 tỷ lệ quan sát thấy polyp và không thấy polyp gần như nhau

Bảng 5. Chu vi và diện tích polyp trên OCTA

\begin{tabular}{|c|c|c|}
\hline $\begin{array}{c}\text { Đặc điếm } \\
\text { polyp }\end{array}$ & TB \pm SD & $\begin{array}{c}\text { Nhỏ nhất - } \\
\text { lớn nhất }\end{array}$ \\
\hline Chu vi polyp & $1,51 \pm 0,65$ & $0,74-3,24$ \\
\hline Diện tích polyp & $0,17 \pm 0,16$ & $0,02-0,57$ \\
\hline
\end{tabular}

Trên OCTA chúng tôi xác định được chu vi polyp trung bình $1,51 \pm 0,65$ và diện tích trung bình $0,17 \pm 0,16$.

Mật độ mạch máu (vessel density) và mật độ tưới máu ở mạng mạch nông của võng mạc tại vị 
trí tân mạch cũng được khảo sát trên hình ảnh OCTA.

Bảng 6. Mật độ mạch máu $(n=24)$

\begin{tabular}{|c|c|c|}
\hline $\begin{array}{c}\text { Tốc độ dòng } \\
\text { chảy }\end{array}$ & $\begin{array}{c}\text { TB } \pm \text { SD } \\
\left(\mathbf{m m} / \mathbf{m m}^{\mathbf{2}}\right)\end{array}$ & $\begin{array}{c}\text { Nhỏ } \text { nhất - } \\
\text { lớn nhất }\end{array}$ \\
\hline Trung tâm & $12,6 \pm 4,8$ & $6,1-25,4$ \\
\hline Quanh trung tâm & $16,4 \pm 3,9$ & $5,7-22,2$ \\
\hline Tống hợp & $16,1 \pm 3,8$ & $5,7-21$ \\
\hline
\end{tabular}

Mật độ trung bình của mạch máu võng mạc ở vùng trung tâm là $12,6 \pm 4,8 \mathrm{~mm} / \mathrm{mm}^{2}$, thấp hơn so với vùng quanh trung tâm (16 \pm $\left.4,6 \mathrm{~mm} / \mathrm{mm}^{2}\right)$.

Bảng 7. Mật độ tưới máu

\begin{tabular}{|c|c|c|}
\hline $\begin{array}{c}\text { Mật độ tưới } \\
\text { máu }\end{array}$ & $\begin{array}{c}\text { TB } \pm \text { SD } \\
(\%)\end{array}$ & $\begin{array}{c}\text { Nhỏ nhất - } \\
\text { Iớn nhất }\end{array}$ \\
\hline Trung tâm & $24,3 \pm 10,6$ & $2,9-45,7$ \\
\hline Quanh trung tâm & $31,3 \pm 7,1$ & $12,4-40,1$ \\
\hline Tống hợp & $30,6 \pm 6,6$ & $12,4-40$ \\
\hline
\end{tabular}

Mật độ tưới máu trung bình ở vùng trung tâm là $24,3 \pm 10,6$, thấp hơn so với vùng quanh trung tâm $\left(31,3 \pm 7,1 \mathrm{~mm} / \mathrm{mm}^{2}\right)$

\section{BÀN LUÂN}

Bệnh mạch máu hắc mạc dạng polyp (PCV) được nghiên cứu nhiều trong những năm gần đây. Trước đây, PCV được coi là thuộc nhóm tân mạch hắc mạc trong thoái hóa hoàng điểm tuổi già. Gần đây các nghiên cứu chỉ ra sự khác biệt giữa hai bệnh lý này. Bệnh thường gặp ở độ tuổi 50 - 65 tuổi, thấp hơn so với thoái hóa hoàng điểm thể già. Nghiên cứu của chúng tôi độ tuổi mắc bệnh là 50-70 tuổi (chiếm 78,6\%) tương ứng với các nghiên cứu trên thế giới. Trước kia PCV được cho là bệnh găp ở nữ, gần đây nghiên cứu chỉ ra rằng tỉ lệ nam/nữ là $4,7 / 1$. Trong nghiên cứu của chúng tôi là 1,5/1.

Trên đáy mắt, xuất hiện nốt sần màu đỏ cam là hình ảnh gợi ý PCV kèm bong biểm mô sắc tố, bong thanh dịch, xuất tiết, xuất huyết. Chúng tôi gặp 20/28 bệnh nhân, đặc biệt dấu hiệu xuất huyết võng mac $23 / 28$ bệnh nhẩn do đa số bệnh nhân đến muộn, khối polyp võ̃, xuất huyết dày che lấp các triệu chứng khác trên đáy mắt.

Phương pháp kỹ thuật chụp mạch OCT (OCTA: Optical Coherence Tomography Angiography) là một phát triển mới nhất trong lĩnh vực chụp mạch máu võng mạc, hắc mạc được phát minh và sáng chế bởi David Huang và Yali ${ }^{5}$. Đây là phương pháp chụp mạch không xâm lấn do không cần tiêm thuốc vì vậy hạn chế được các tai biến liên quan đến tiêm thuốc. Kỹ thuật chụp nhanh và rõ nét các dòng chảy trong lòng mạch máu võng mạc, chụp OCTA sẽ là kỹ thuật hữu ích giúp các bác sỹ nhãn khoa quản lý các bệnh lý mạch máu võng mạc và hắc mạc. Chụp OCTA đã được áp dụng để đánh giá tân mạch hắc mạc trong bệnh thoái hóa hoàng điểm tuổi già, bệnh hắc võng mạc trung tâm thanh dịch, bệnh cận thị. Chụp mạch OCT là một phương pháp mới được phát triển cho phép chúng ta hình dung tuân hoàn hắc mạc mà không cân sử dụng thuốc cản quang. Phương pháp này đã được áp dụng cho các bệnh lý mạch máu võng mạc khác nhau và được báo cáo rẳng OCTA rất hữu ích để phát hiện các tổn thương mạch máu bất thường ${ }^{6}$.

Trong nghiên cứu hiện tại của chúng tôi, tất cả các BVN và các polyp được OCTA phát hiện nằm giữa biểu mô sắc tố và màng Bruch, kết quả của chúng tôi tương thích với một báo cáo trước đó. Huang và cộng sự chia BVN thành các loại hình dạng: thân cây, câuu thận và các mấu hình que. Nhưng nhìn chung, các BVN được biểu hiện dưới dang tín hiêu tần số cao trên OCTA và có thể được xác định một cách dễ dàng. Trong nghiên cứu của chúng tôi ICGA phát hiện BVN

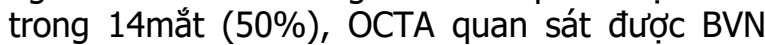
trong 16 mắt $(57,1 \%)$. Nghiên cứu đã xác định ba loại mẫu BVN khác nhau dựa trên các đặc điểm hình thái trên OCTA. Các mẫu BVN không chỉ cho thấy các biểu hiện lâm sàng khác nhau, mà còn có thể cung cấp thông tin có giá trị về giải phẫu. Khi công nghệ OCTA đang được áp dụng rộng rãi để phát hiện các cấu trúc tân mạch, BVN đã được xác định $90 \%$ các trường hợp PCV, nhưng các polyp đôi khi không được xác định rõ. Hình thái của BVN có thể được phân biệt thành 3 loại tương ứng tỉ lệ: $14,3 \%$ loại 1 , $28,6 \%$ loại $2,14,3 \%$ loại 3 , có $12 / 28$ mắt không quan sát được BVN trên OCTA (chiếm 42,9\%) do bệnh nhân phát hiện bệnh muộn khi đến khám xuất huyết dưới võ̃ng mạc rộng che lấp khiến quan sát tổn thương gặp khó khăn.

Trong nghiên cứu chúng tôi một số trường hợp polyp và $B V N$ quan sát rõ trên $O C T-H D$ và trên OCTA sẽ không tiến hành chụp ICGA; trường hợp quan sát trên OCTA không rõ polyp và $B V N$ đồng thời nghi ngờ trên OCT-HD chúng tôi tiến hành chụp ICG để chẩn đoán chính xác.

\section{KẾT LUẬN}

Bệnh mạch máu hắc mạc dạng polyp (PCV) mới được nghiên cứu trong những năm gần đây, nhờ sự tiến bộ của phương tiện chẩn đoán như chụp cắt lớp võng mạc (OCT), chụp mạch huỳnh quang (FA), chụp xanh indocyanine (ICG), chụp mạch OCT (OCTA). Triệu chứng lâm sàng hay gặp: nhìn mờ, ám điểm, nhìn méo hình... Soi đáy 
mắt hay gặp nốt sần màu vàng cam, xuất huyết, bong thanh dịch... Trên OCT thấy dấu hiệu bong biểu mô sắc tố dạng vòm, dấu hiệu hai lớp tương ứng với hình ảnh mạng mạch nhánh (BVN) trên OCTA. Hình thái của BVN có thể được phân biệt thành 3 loại, việc xác định hình thái học của BVN có tương quan với biểu hiện lâm sàng và tiên lượng ở bệnh nhân PCV. OCTA có thể phát hiện polyp và $B V N$ và độ nhạy phát hiện của nó thấp hơn đối với polyp và cao hơn đối với BVN so với phát hiện của ICGA. ICGA xâm lấn nhiều hơn có thể được dành cho các trường hợp ít hơn với OCTA âm tính nhưng nghi ngờ PCV cao.Hạn chế của việc phát hiện polyp bằng OCTA bao gồm bong biểu mô sắc tố cao và polyp nhỏ và ICGA nên được thực hiện để xác định chẩn đoán trong các trường hợp nghi ngờ PCV trên lâm sàng.

\section{TÀI LIÊU THAM KHẢO}

1. Ciardella AP, Donsoff IM, Huang SJ, Costa DL, Yannuzzi LA. Polypoidal choroidal vasculopathy. Survey of ophthalmology. 2004; 49(1):25-37.

2. Cackett $P$, Wong $D$, Yeo I. A classification system for polypoidal choroidal vasculopathy. Retina. 2009;29(2):187-191.

3. Seong S, Choo HG, Kim YJ, et al. Novel Findings of Polypoidal Choroidal Vasculopathy via Optical Coherence Tomography Angiography. Korean journal of ophthalmology : KJO. 2019;33(1):54-62.

4. Kawamura A, Yuzawa M, Mori R, Haruyama M, Tanaka K. Indocyanine green angiographic and optical coherence tomographic findings support classification of polypoidal choroidal vasculopathy into two types. Acta ophthalmologica. 2013;91(6):e474-481.

5. Tomiyasu T, Nozaki M, Yoshida M, Ogura Y. Characteristics of Polypoidal Choroidal Vasculopathy Evaluated by Optical Coherence Tomography Angiography. Investigative ophthalmology \& visual science. 2016;57(9):OCT324-330.

6. Chaikitmongkol V, Cheung CMG, Koizumi H, Govindahar V, Chhablani J, Lai TYY. Latest Developments in Polypoidal Choroidal Vasculopathy: Epidemiology, Etiology, Diagnosis, and Treatment. Asia-Pacific journal of ophthalmology (Philadelphia, Pa.). 2020;9(3):260-268.

7. Hwang DK, Yang CS, Lee FL, Hsu WM. Idiopathic polypoidal choroidal vasculopathy. Journal of the Chinese Medical Association : JCMA. 2007;70(2):84-88.

\section{KẾT QUẢ ĐIÎ̀U TRI BỔ TRỢ TRƯớC PHÁC Đồ HÓA CHẤT KẾT HỢP TRASTUZUMAB VÀ PERTUZUMAB TRÊN UNG THƯ VÚ CÓ HER2-NEU DƯO'NG TÍNH}

\section{TÓM TẮT}

Điều trị hóa chất kết hợp các thuốc kháng HER2neu đã trở thành tiêu chuẩn trong điều trị ung thư vú có HER2-neu dương tính trên thế giới. Tại Việt Nam, Trastuzumab được sử dụng từ năm 2006 còn Pertuzumab mới được đưa vào sử dung gân đây. Nghiên cứu mô tả hồi cứu kết hợp tiến cứu đánh giá kết quả điều tri bổ trơ trước phác đồ hóa chất kết hợp Trastuzumab và Pertuzumab trên bệnh nhân ung thư vú có HER2-neu dương tính tại bênh viên $\mathrm{K}$ từ 1/2018 đến 4/2021. Kết quả nghiên cứu trên 20 bệnh nhân cho thấy tỉ lệ đáp ứng toànbộ trên lâm sàng đạt 95,0\%, có một trường hợp bệnh tiến triển. Đáp ứng mô hoc hoàn toàn toàn bô cá u và hach (tpCR) đat $80,0 \%$. Không có mối liên quan giữa tỷ lệ tpCR và các yếu tố tuổi, giai đoan u, giai đoạn hạch, type mô bệnh học, độ mô học, tình trạng nội tiết, Ki67 và phác đồ hóa chất. Phác đồ dung nạp tốt, không có trướng hợp nào trì hoãn hoặc dừng điểu trị do độc tính.

Tư khóa: hóa chất bổ trợ trước, pertuzumab, ung thư vú HER2-neu dương tính

*Bênh viên K

Chịu trách nhiệm chính: PhùngThị Huyền

Email: phungthihuyen@gmail.com

Ngày nhân bài: 10.9.2021

Ngày phản biên khoa hoc: 1.11.2021

Ngày duyệt bài: 12.11.2021
Phùng Thị Huyền*

\section{SUMMARY}

EFFICACY OF NEOADJUVANT THERAPY WITH

CHEMOTHERAPY COMBINED WITH TRASTUZUMAB AND PERTUZUMAB IN PATIENTS WITH HER2-NEU POSITIVE BREAST CANCER

HER2-neu targeted therapy in combination with chemotherapy has been the standard treatment for HER2-neu positive cancer in the world. In Vietnam, Trastuzumab has been used since 2016 while Pertuzumab has just recently been used. This is a descriptive cohort study evaluating the efficacy of neoadjuvant Trastuzumab and Pertuzumab in combination with chemotherapy for HER2-neu positive breast cancer at K hospital from 01/2018 to 04/2021. The results on 20 patients showed that the clinical overall response rate was $95.0 \%$, with only one patient progressed. Total pathological complete response (tpCR) rate was $80.0 \%$. There were no associations between tpCR and age, tumor and nodal stage, histological type and grade, ER, PR, Ki67 status and chemotherapy regimen. The regimens were well tolerated and no patients had treatment delay or interruption due to toxicities.

Keywords: neoadjuvant, Pertuzumab, HER2-neu positive breast cancer

\section{I. ĐĂT VẤN ĐỀ}

Ung thư vú là bệnh ung thư có tỉ lệ mắc cao 